The Astrophysical Journal, 555:178-190, 2001 July 1

(C) 2001. The American Astronomical Society. All rights reserved. Printed in U.S.A.

\title{
FILAMENTARY STRUCTURE AND HELICAL MAGNETIC FIELDS IN THE ENVIRONMENT OF A STARLESS DENSE CORE
}

\author{
E. FALGARONE \\ Radioastronomie, Ecole Normale Supérieure, 24 rue Lhomond, 75005 Paris, France \\ J. PeTY \\ IRAM, 300 rue de la Piscine, 38406 Saint Martin d'Hères, France \\ AND \\ T. G. PHILliPS \\ California Institute of Technology, Pasadena, CA 91125 \\ Received 2000 November 22; accepted 2001 February 22
}

\begin{abstract}
The environment of L1512, a starless dense core, has been mapped at high angular resolution in the ${ }^{12} \mathrm{CO}(J=2-1)$ line over more than $1 \mathrm{pc}$, with a few positions observed in the ${ }^{12} \mathrm{CO}(J=3-2)$ and $(J=4-3)$ lines. The gas outside the dense core is structured in several filaments, roughly 1 pc long and $\sim 0.1 \mathrm{pc}$ thick, converging at the dense core position. Small longitudinal $\left(\sim 1 \mathrm{~km} \mathrm{~s}^{-1} \mathrm{pc}^{-1}\right) \mathrm{but}^{-1 a r g e}$ transverse (up to $8 \mathrm{~km} \mathrm{~s}^{-1} \mathrm{pc}^{-1}$ ) velocity gradients are observed. Remarkably, the transverse gradients can be seen to change sign periodically, along at least one of the filaments. Thus, there are oscillations in the toroidal velocity within the filaments, which may be a signature of a magnetohydrodynamic instability developing in filaments permeated by a helical magnetic field. In the case of L1512, according to the analysis of Fiege \& Pudritz, the growth rate of the instability is low, corresponding to a timescale of the order of 1 Myr. We deduce from the wavelength of the oscillations that the toroidal component of the magnetic field dominates the poloidal component. The toroidal component helps confine the filaments, which are not otherwise confined by self-gravity $\left(m / m_{\text {vir }} \sim 0.2\right)$, by the pressure of the galactic $H$ I layer, or by external turbulent pressure. We find that the velocity gradients in the vicinity of the dense core provide an estimate for an upper limit to the accretion rate onto the dense core of $\dot{M}=4 \times 10^{-6}$ $M_{\odot} \mathrm{yr}^{-1}$. For the gas characteristics in the filaments, we find that a broad range of density and temperature is allowed for the gas, from $n_{\mathrm{H}_{2}}=2 \times 10^{3} \mathrm{~cm}^{-3}$ for the coldest case $\left(T_{k}=20 \mathrm{~K}\right)$ down to $n_{\mathrm{H}_{2}}=$ $180 \mathrm{~cm}^{-3}$ for the warmest $\left(T_{k}=250 \mathrm{~K}\right)$.

Subject headings: ISM: clouds - ISM: magnetic fields - ISM: molecules
\end{abstract}

\section{INTRODUCTION}

It is by now well established observationally that massive molecular clouds are close to virial balance between selfgravity and the nonthermal energy contained in their turbulent motions (Dame et al. 1986; Scoville et al. 1987; Solomon et al. 1987; Falgarone \& Pérault 1987). A large fraction of molecular clouds, though, are not gravitationally bound, in particular the smallest, least massive structures (Falgarone, Puget, \& Pérault 1992), and they are likely confined by an external pressure, possibly of turbulent origin (Bertoldi \& McKee 1992; Vázquez-Semadeni et al. 2000). It is therefore to be expected that the criterion for the development of the gravitational instability is not the Jeans criterion, which involves only local and scale-free quantities (the density and temperature of the gas), but rather that the formation of small-scale, self-gravitating structures, such as dense cores, and the eventual collapse of stars within these cores are governed by processes rooted in their large-scale environment.

However, the interplay of turbulence and its dissipation with the evolution of molecular clouds is complex. Recent numerical simulations of supersonic turbulence with gravity have demonstrated the rapid formation of dense selfgravitating structures resulting from shocks and shock interactions (Klessen, Heitsch, \& Mac Low 2000). These simulations confirm that turbulence prevents global collapse, as had been shown analytically by Bonazzola et al. $(1987,1992)$. Other lines of investigation have been devel- oped. Myers \& Lazarian (1998) propose that dense cores form in turbulent cooling flows driven by local gradients of turbulent pressure. The impact of the timescales involved in the scale-dependent turbulent pressure and, more importantly, in its gradients, must be addressed before such a scenario can be validated. In molecular clouds, the neutrals and the ions are not closely coupled at small scales (and short timescales), and ion-neutral friction in magnetohydrodynamic (MHD) shocks or in MHD waves may generate an important dissipation (Kulsrud \& Pearce 1969; Flower \& Pineau des Forêts 1998). The ion-neutral drift has not been taken into account yet in numerical simulations of MHD turbulence that allow estimates of the timescales for energy dissipation (Ostriker, Gammie, \& Stone 1999; Mac Low 1999). Finally, viscous processes (elastic neutral-neutral collisions) occur in the regions of largest shear of the velocity field, localized, for instance, at the boundary of coherent vortices. Laboratory experiments of incompressible turbulence (Douady, Couder, \& Brachet 1991; Cadot, Douady, \& Couder 1995) suggest that these vortices have a crosssectional radius between the inner Kolmogorov scale $l_{d}$ (or the dissipation scale) and the Taylor microscale, $l_{d}^{1 / 3} L^{2 / 3}, L$ being the integral scale. Numerical simulations of compressible turbulence cannot properly describe the fluid properties so close to the dissipation scale (Porter, Pouquet, \& Woodward 1994), but the fraction of the turbulent energy in the solenoidal part (incompressible) of the velocity field is significant. The Kolmogorov scale in molecular clouds is 
estimated to be of the order of $10 \mathrm{AU}$, and therefore is orders of magnitude smaller than size scales accessible to current observations. Dissipation occurring at these small scales can therefore be studied only with statistical tools (Lis et al. 1996; Pety 1999) or chemical signatures (Joulain et al. 1998).

With the expectation of shedding light on the processes leading to the formation of dense cores within molecular clouds and of analyzing the dynamics of the gas in the vicinity of such cores, maps of the environment of three dense cores without embedded stars have been made in the two lowest transitions of $\mathrm{CO}$ and isotopes over fields of a few square arcminutes around low-mass dense cores (Falgarone et al. 1998). The results were as follows. (1) The cores have an internal velocity dispersion much smaller than that of their environment over a similar size scale. (2) The core environment in all three fields is highly structured and exhibits conspicuous filaments with different properties in each field. In the L1512 field, there are only a few bright filaments, while in another field (in the Polaris Flare), the filaments are more numerous, less organized spatially, thinner, and smaller. (3) The gas component outside the dense cores has a high ${ }^{12} \mathrm{CO} /{ }^{13} \mathrm{CO}$ ratio. (4) The ${ }^{12} \mathrm{CO}$ line shapes are non-Gaussian, and it was shown that the line wing emission originates primarily in the filamentary structures. A mosaic of 13 fields has been made with the Plateau de Bure interferometer in the ${ }^{12} \mathrm{CO}(J=1-0)$ line in the environment of the dense core in the Polaris Flare (Pety \& Falgarone 2001, in preparation). The angular resolution of this map is $3.8 \times 3$.".5, or $500 \mathrm{AU}$, at the distance of the flare $(d=150 \mathrm{pc})$. Extremely large transverse velocity gradients have been measured in two of the filaments: velocity differences of $3.5 \mathrm{~km} \mathrm{~s}^{-1}$ over $1500 \mathrm{AU}$, or $\nabla v \sim 450 \mathrm{~km} \mathrm{~s}^{-1}$ $\mathrm{pc}^{-1}$, implying rotational periods as small as $10^{4} \mathrm{yr}$.

The work here is a follow-up of this study, carried out on the field surrounding the core L1512. Our goals are to further characterize the density and temperature, as well as the dynamics, of the gas in the filaments that make up the core environment. To do so, we observed the line wing emission with a high signal-to-noise ratio in the ${ }^{12} \mathrm{CO}$ $(J=3-2)$ and $(J=4-3)$ transitions to better constrain the excitation of the gas in the filaments, and we made a ${ }^{12} \mathrm{CO}$ $(J=2-1)$ large-scale map in the on-the-fly mode. A statistical analysis of the turbulent velocity field, in particular that of its non-Gaussian features, has been conducted in the $1 \mathrm{pc}$ field around L1512 along the lines developed in Lis et al. (1996). It allows the localization of the regions of largest vorticity (Pety 1999; Pety \& Falgarone 2001, in preparation).

In $\S 2$, we describe the observations. In $\S 3$, we present the observational results regarding the velocity field in the filaments and their density and temperature. In $\S 4$, we discuss the possible heating sources for the detected warm gas, provide a tentative interpretation of the data invoking a helical magnetic field to confine the filaments (Fiege \& Pudritz 2000a, 2000b), and give the characteristics of the gas inflow onto the dense core.

\section{OBSERVATIONS}

The selected dense core L1512 is a small (size $\sim 0.1 \mathrm{pc}$ ), nearby, low-mass dense core with no signpost of star formation. It is located at the edge of the Taurus-Auriga-Perseus complex in a low average column density region at the parsec scale $\left(N_{\mathrm{H}_{2}}\right.$ of a few $\left.10^{20} \mathrm{~cm}^{-2}\right)$. This is deduced from the low angular resolution ${ }^{12} \mathrm{CO}(J=1-0)$ observations of Ungerechts \& Thaddeus (1987). The fact that the dense core is surrounded by an almost transparent environment helps in isolating the gas directly connected to the core and in minimizing confusion with unrelated components. The local $\mathrm{H}_{2}$ density within the dense core is deduced from multitransition observations of several molecules (CS, $\mathrm{HC}_{3} \mathrm{~N}, \mathrm{NH}_{3}$, and $\mathrm{C}_{3} \mathrm{H}_{2}$ ) and is in the range $3 \times 10^{4}-10^{5}$ $\mathrm{cm}^{-3}$. The $\mathrm{C}_{3} \mathrm{H}_{2}$ lines in this core are among the narrowest ever observed, but their small line width cannot be accounted for by thermal motions alone, as is also the case for the $\mathrm{NH}_{3}$ line widths. The average gas temperature in the core is estimated to be $12 \mathrm{~K}$, and the nonthermal contribution to the observed motions is only $\sigma_{\mathrm{NT}}=0.038 \mathrm{~km} \mathrm{~s}^{-1}$. L1512 is one of the least centrally condensed cores detected in the submillimeter continuum by Ward-Thompson et al. (1994) and does not have any spectral signature of infall motion toward a central object (Gregersen \& Evans 2000).

Observations were performed with the $10.4 \mathrm{~m}$ antenna of the Caltech Submillimeter Observatory (CSO) in 1997 January and December for the high-frequency lines ${ }^{12} \mathrm{CO}$ $(J=3-2)$ and $(J=4-3)$ and in 1997 December and 1998 November for the ${ }^{12} \mathrm{CO}(J=2-1)$ map. The single sideband (SSB) system temperature, including the effects of the Earth's atmosphere, was $\sim 2000 \mathrm{~K}$ at $461 \mathrm{GHz}$ and $\sim 700$ $\mathrm{K}$ at $346 \mathrm{GHz}$. The zenith opacity at $220 \mathrm{GHz}$ was about 0.05 during the high-frequency observations. The SSB system temperature was $\sim 270 \mathrm{~K}$ at $230 \mathrm{GHz}$, with a zenith opacity of about 0.1 . The efficiencies, measured on small planets at intermediate zenith angles, are $0.55,0.75$, and 0.8 at $461 \mathrm{GHz}, 346 \mathrm{GHz}$, and $230 \mathrm{GHz}$, respectively. The data were taken with an acousto-optical spectrometer whose effective resolution varied between 100 and $150 \mathrm{kHz}$. Frequency calibration was performed regularly. The $100 \mathrm{kHz}$ resolution corresponds to $0.065 \mathrm{~km} \mathrm{~s}^{-1}$ at $461 \mathrm{GHz}$. The angular resolution (half-power beamwidth) of the telescope is $16^{\prime \prime}, 22^{\prime \prime}$, and $28^{\prime \prime}$, at the three frequencies, respectively. Pointing was checked either on planets or on the line emission of protoplanetary sources such as CRL 618. The intensity calibration was performed with the classical hot-cold load measurements. The high-frequency spectra were obtained after long integrations in the position-switching mode. The ${ }^{12} \mathrm{CO}(J=2-1)$ map was constructed with the on-the-fly technique at a speed of $2^{\prime \prime} \mathrm{s}^{-1}$, with a dump of the data every $8 \mathrm{~s}$ (or one-half beam). The size of individual scans between two comparison measurements was $360^{\prime \prime}$ in R.A. The whole map has been observed twice and contains 8750 spectra with an average $\mathrm{rms}$ noise level, for a resolution of $100 \mathrm{kHz}$, of about $0.2 \mathrm{~K}$. The area of the ${ }^{12} \mathrm{CO}$ $(J=2-1)$ map $\left(24^{\prime} \times 26^{\prime}\right)$ is therefore about 12 times larger than the IRAM $30 \mathrm{~m}$ map $\left(5^{\prime} \times 10^{\prime}\right)$ and covers an area of $1.2 \mathrm{pc} \times 1 \mathrm{pc}$ around the dense core.

\section{OBSERVATIONAL RESULTS}

\subsection{Morphology and Mass Distribution}

The ${ }^{12} \mathrm{CO}(J=2-1)$ line-integrated intensity map is displayed in Figure $1 a$. The region of the ${ }^{12} \mathrm{CO}$ peak (about $\left.4^{\prime} \times 4^{\prime}\right)$ corresponds to the dense core as traced in the $\mathrm{C}^{18} \mathrm{O}$ line (see Fig. 2 of Falgarone et al. 1998, hereafter F98). The distribution of the ${ }^{12} \mathrm{CO}$ line emission is highly anisotropic and is concentrated in a network of filamentary structures. These filaments are identified with numbers from 1 to 6 in 


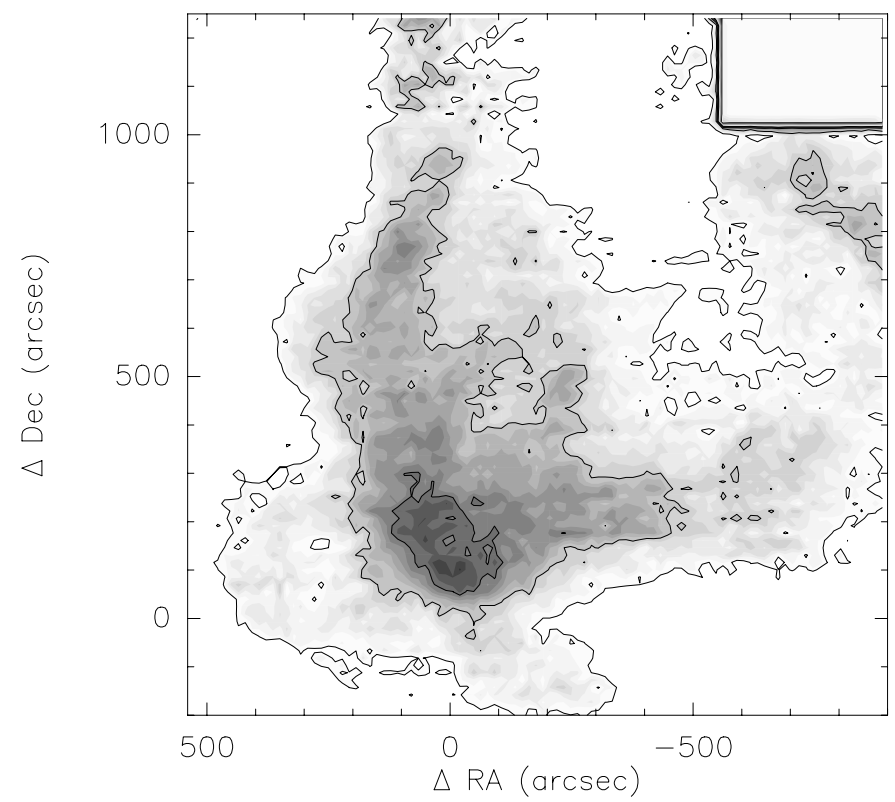

FIG. $1 a$

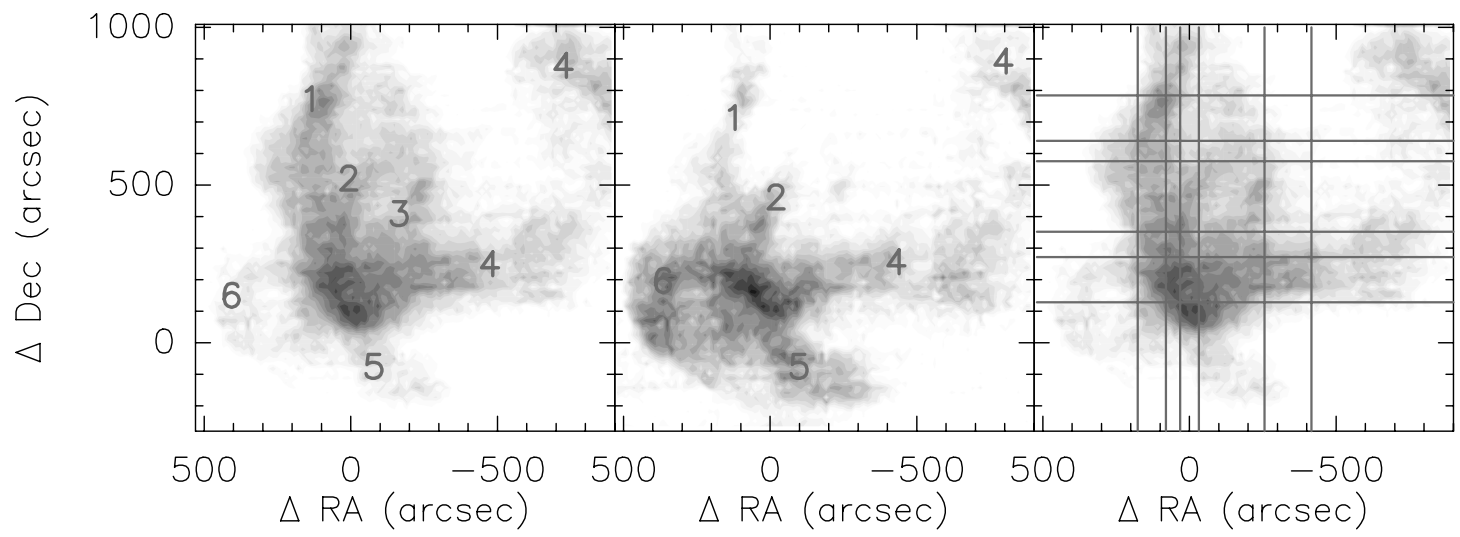

FIG. $1 b$

FIG. 1.- (a) Map of the integrated line intensity of the ${ }^{12} \mathrm{CO}(J=2-1)$ line observed in the on-the-fly mode at the CSO. The contour levels are 1 , 2 , and 3 $\mathrm{K} \mathrm{km} \mathrm{s}^{-1}$. The $(0,0)$ position is $\alpha(1950)=05^{\mathrm{h}} 00^{\mathrm{m}} 54.5, \delta(1950)=32^{\circ} 36^{\prime}$. The offsets are in arcseconds. The dense core, barely visible in the ${ }^{12} \mathrm{CO}$ emission, is centered at $\left(0^{\prime \prime}, 180^{\prime \prime}\right)$ and has a diameter of about $200^{\prime \prime}$, or $0.15 \mathrm{pc}$. (b) Maps of the ${ }^{12} \mathrm{CO}(J=2-1)$ line intensity integrated over different velocity intervals (whole line [left and right panels] or [7.3, 8.1] $\mathrm{km} \mathrm{s}^{-1}$ [center panel]) in order to individualize the six filaments discussed in the text and display the cuts corresponding to the space-velocity diagrams of Figs. 3 and 4.

Figure $1 b$, which shows the ${ }^{12} \mathrm{CO}(J=2-1)$ emission integrated over different velocity intervals. Three of these structures $(1,4$, and 5$)$ may extend beyond the limits of the map and two of them (1 and 4$)$ are as long as $0.75 \mathrm{pc}$, with a thickness (at half intensity) varying between 0.08 and $0.2 \mathrm{pc}$. These filaments all converge in projection toward the location of the dense core centered at offset position $\left(0^{\prime \prime}, 180^{\prime \prime}\right)$.

A histogram of the line-integrated brightness distribution has been built. The positions above $W(\mathrm{CO})=3 \mathrm{~K} \mathrm{~km} \mathrm{~s}^{-1}$ belong to the dense core region (see Fig. 1a), those with $W(\mathrm{CO})$ between 1 and $3 \mathrm{~K} \mathrm{~km} \mathrm{~s}^{-1}$ belong to the filaments, and those between 0.1 and $1 \mathrm{~K} \mathrm{~km} \mathrm{~s}^{-1}$ to the background $\left(0.1 \mathrm{~K} \mathrm{~km} \mathrm{~s}^{-1}\right.$ is the $3 \sigma$ level for the integrated emission). Although the filaments and the background fill approximately the same fractional area in the map, the mass fraction in the filaments, estimated from $W(\mathrm{CO})$, is $75 \%$ of the total mass in the map, the dense core apparently contributing less than $1 \%$, which is to be expected from the large optical depth of the ${ }^{12} \mathrm{CO}(J=2-1)$ line in the direction of the dense core. If the $\mathrm{CO}$ optical depth is taken into account, the mass fraction in the core becomes about one half, as explained below.

The brightness contrast in ${ }^{12} \mathrm{CO}$ between the core and its environment is only about 4 to 1 , as seen above, while the contrast in column density, as derived from the $\mathrm{C}^{18} \mathrm{O}$ $(J=1-0)$ line for the core and the ${ }^{12} \mathrm{CO}(J=2-1)$ line for the environment, is $\approx 20$ to 1 . The $\mathrm{C}^{18} \mathrm{O}(J=1-0)$ line intensity in the direction of the core is $2 \mathrm{~K}$ and the line width is $0.2 \mathrm{~km} \mathrm{~s}^{-1}$ (F98). It corresponds to an $\mathrm{H}_{2}$ column density $N_{\mathrm{H}_{2}}$ of $2 \times 10^{22} \mathrm{~cm}^{-2}$ for $n_{\mathrm{H}_{2}}=3 \times 10^{4} \mathrm{~cm}^{-3}$, while the ${ }^{12} \mathrm{CO}(J=2-1)$ integrated line intensity of 1 to 3 $\mathrm{K} \mathrm{km} \mathrm{s}^{-1}$ in the core environment corresponds to $N_{\mathrm{H}_{2}} \approx$ $10^{21} \mathrm{~cm}^{-2}$, assuming $N_{\mathrm{H}_{2}} / W(\mathrm{CO})=2.5 \times 10^{20} \mathrm{~cm}^{-2}(\mathrm{~K}$ $\left.\mathrm{km} \mathrm{s}^{-1}\right)^{-1}$ for the ${ }^{12} \mathrm{CO}(J=1-0)$ line and an $R(2-1 / 1-0)$ brightness ratio of 0.65 (F98). The $\mathrm{H}_{2}$ mass in the dense core, derived from the above estimate of the $\mathrm{H}_{2}$ column density, is $6 M_{\odot}$. The total mass of gas traced by ${ }^{12} \mathrm{CO}$ $(J=2-1)$ within the $1.2 \mathrm{pc} \times 1 \mathrm{pc}$ field is $10 M_{\odot}$, as 
deduced from the $(J=2-1)$ integrated line intensity over the whole map $\left[W(\mathrm{CO})=1.3 \mathrm{~K} \mathrm{~km} \mathrm{~s}^{-1}\right]$ and the conversion factor and line ratios quoted above.

\subsection{The Velocity Field}

\subsubsection{Filamentary Structure of the Line Wing Emission}

In the two lowest rotational transitions, the ${ }^{12} \mathrm{CO}$ to ${ }^{13} \mathrm{CO}$ line ratios were found to vary significantly across the line profiles (F98). For this reason, in this work the ${ }^{12} \mathrm{CO}$ line emission of L1512 was divided into the line core and line wing emission on the sole basis of the ${ }^{12} \mathrm{CO}$ to ${ }^{13} \mathrm{CO}$ line ratios. The line core emission arises in the velocity interval $[6.5,7.3] \mathrm{km} \mathrm{s}^{-1}$ and corresponds to the velocity coverage of the dense core (i.e., gas-bright in ${ }^{13} \mathrm{CO}$ and $\mathrm{C}^{18} \mathrm{O}$ ). In this velocity interval the ${ }^{12} \mathrm{CO}$ to ${ }^{13} \mathrm{CO}$ line ratios are close to unity at the peaks. For the line wing emission, over the velocity range $[7.3,8.1] \mathrm{km} \mathrm{s}^{-1}$, the ${ }^{12} \mathrm{CO}$ to ${ }^{13} \mathrm{CO}$ ratios are much higher, from a few up to $\sim 20$. The gas, which has a high ${ }^{12} \mathrm{CO} /{ }^{13} \mathrm{CO}$ ratio, likely also radiates in the velocity range of the line core emission but cannot be disentangled from it in this velocity range. It is easily traced by the ${ }^{12} \mathrm{CO}$ lines, but studying it with ${ }^{13} \mathrm{CO}$ emission would require very long integrations. In the following, we rely on the findings of F98, namely that the space-velocity structure of the gas emitting the ${ }^{12} \mathrm{CO}$ line allows a large photon-escape probability. We therefore stress the fact that the ${ }^{12} \mathrm{CO}$ line, in velocity intervals avoiding that of the dense core, traces the bulk of the molecular material.

Maps of the ${ }^{12} \mathrm{CO}(J=2-1)$ emission integrated over velocity bins of width $0.5 \mathrm{~km} \mathrm{~s}^{-1}$ are displayed in Figure 2 . They illustrate the complex structure of the gas that does not belong to the dense core, i.e., gas farther than $\pm 2^{\prime}$ from offset $\left(0^{\prime \prime}, 180^{\prime \prime}\right)$ at any velocity, or gas within $\pm 2^{\prime}$ of offset $\left(0^{\prime \prime}, 180^{\prime \prime}\right)$ in the velocity range $[7.3,8.1] \mathrm{km} \mathrm{s}^{-1}$. The six filaments identified in Figure $1 b$ can be recognized on the channel maps. Filament 1 is almost straight and extends up to the edge of the present map (i.e., over $900^{\prime \prime}$, or $0.7 \mathrm{pc}$, at the distance of the source, $d=150 \mathrm{pc}$ ). Filaments 2 and 3 are much shorter and are best visible in the velocity ranges
$[7.3,7.5] \mathrm{km} \mathrm{s}^{-1}$ and [7.1, 7.3] $\mathrm{km} \mathrm{s}^{-1}$, respectively. Filament 4 is elongated in the east-west direction and likely forms a loop with the emission in the northwest corner of the map. Filaments 5 , in the south (brighter at $[7.5,7.7] \mathrm{km}$ $\mathrm{s}^{-1}$ ), and 6 , in the east, may be connected to each other. Interestingly, the dense core is located at the point of convergence of all these filaments. It would seem unlikely that this convergence is due only to a projection effect.

As visible in the channel maps, these elongated filaments also emit in the velocity range of the line core, which confirms that the so-called line core emission is a mixture of core and wing emission. The spatial distribution of the line wing emission (usually much weaker than the line core emission) is visible in the position-velocity diagrams of Figure 3. It appears as a weak emission at velocities greater than $7.3 \mathrm{~km} \mathrm{~s}^{-1}$. It is not necessarily associated with line core emission (e.g., the southernmost region in most of the declination cuts and the westernmost regions in the R.A. cuts). It also appears as the extension of an east-west filamentary structure (cut in R.A. at $\delta$ decl. $=272^{\prime \prime}$ ).

\subsubsection{Velocity Gradients}

Figure 2 shows that there is a large-scale gradient of about $1 \mathrm{~km} \mathrm{~s}^{-1} \mathrm{pc}^{-1}$ in the northwest-southeast direction. The space-velocity diagrams of Figures 3 and 4 display the most characteristic patterns observed in the field. They illustrate the variety of the velocity gradients (i.e., their spatial uniformity or, in other cases, their sharp variations in space). In these diagrams, the gradients appear as deviations of the pattern of $\mathrm{CO}$ emission from the vertical direction $(v=$ constant). Their behaviors, displayed in Figures 3 and 4 are complex. The main properties of the velocity gradients are the following:

Longitudinal gradients $\left(\sim \pm 1 \mathrm{~km} \mathrm{~s}^{-1} \mathrm{pc}^{-1}\right)$ are found in the two main filaments (Figs. $3 b$ and $4 b$ are cuts along filaments 1 and 4, respectively). These longitudinal gradients are small but uniform over $400^{\prime \prime}$ or more, i.e., a size scale greater than $0.3 \mathrm{pc}$. Gradients are significantly larger at small scales. It is remarkable that many of these gradients
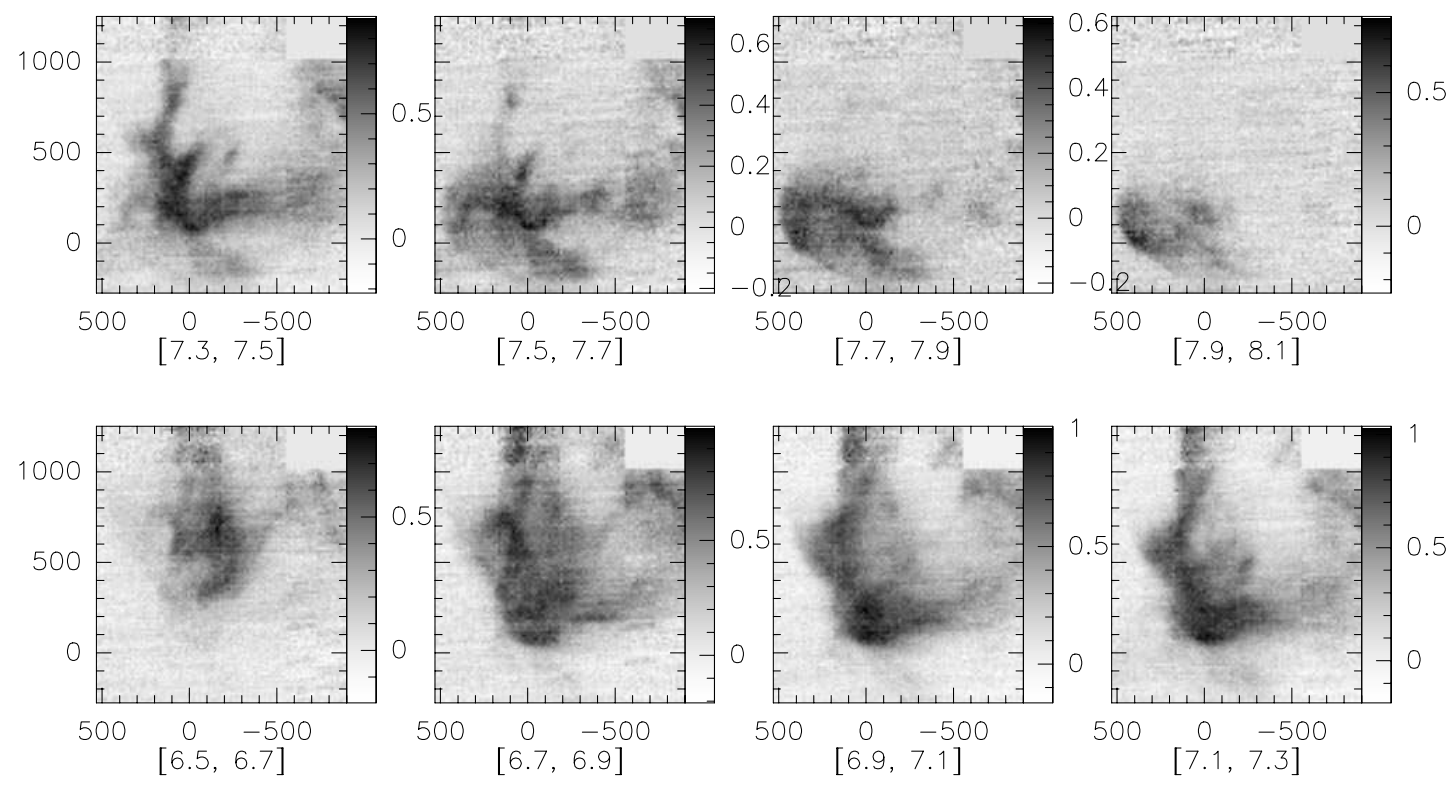

FIG. 2.-Maps of ${ }^{12} \mathrm{CO}(J=2-1)$ intensity integrated over velocity bins of $0.5 \mathrm{~km} \mathrm{~s}^{-1}$, shown below each panel. The intensity scale is different for each map and is provided at the right edge of each panel in $\mathrm{K} \mathrm{km} \mathrm{s}^{-1}$. 
a) $\delta \mathrm{RA}=176 \mathrm{Arcsec}$

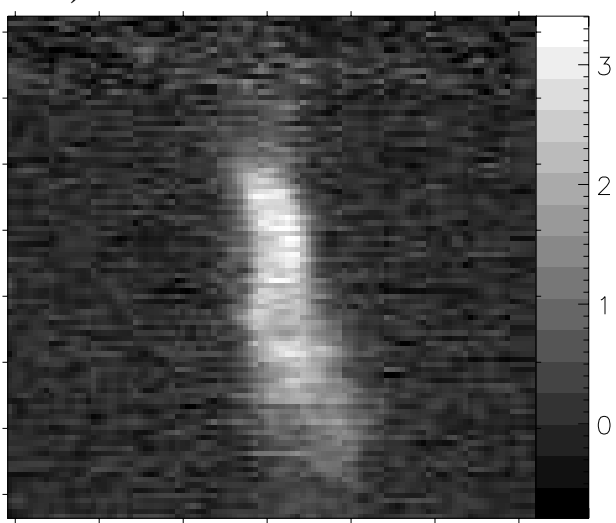

c) $\quad \delta \mathrm{RA}=32 \operatorname{Arcsec}$

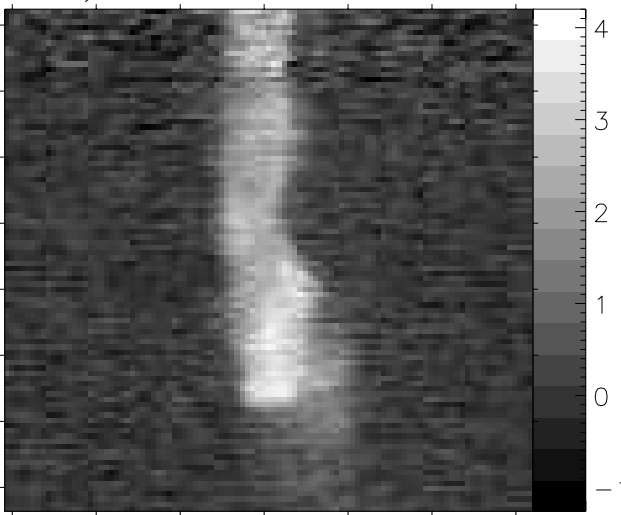

e) $\quad \delta \mathrm{RA}=-256 \operatorname{Arcsec}$

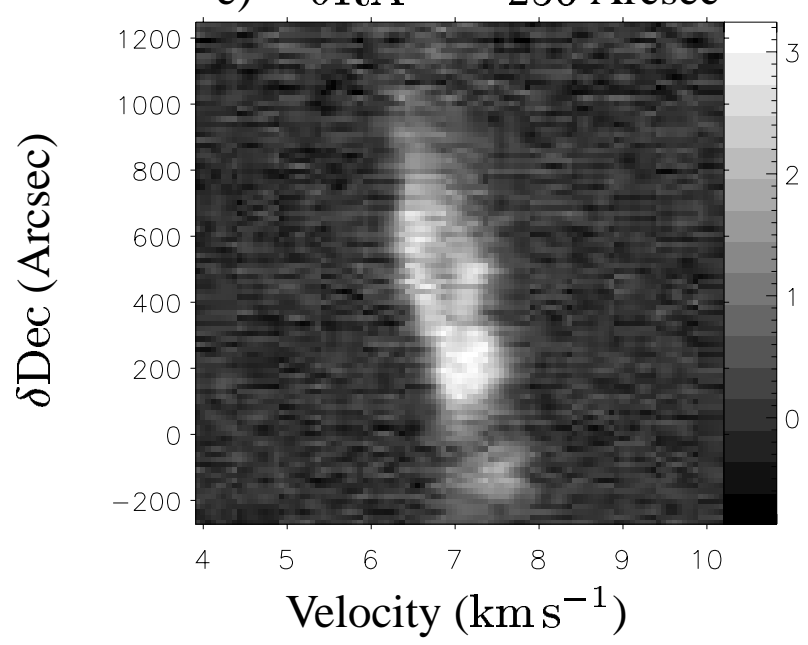

b) $\quad \delta \mathrm{RA}=80 \operatorname{Arcsec}$

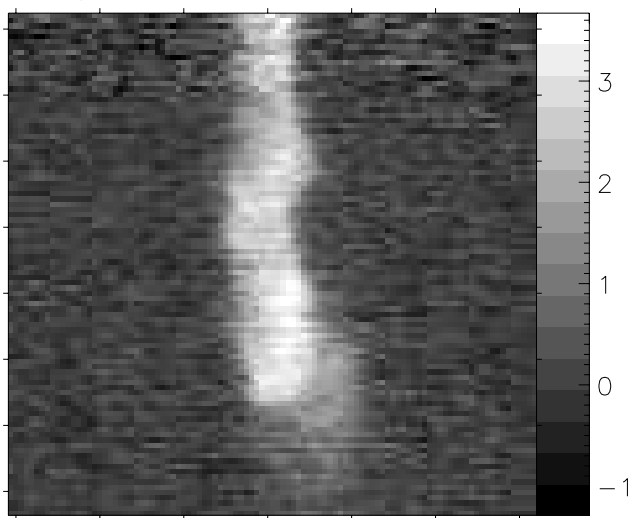

d) $\delta \mathrm{RA}=-32 \operatorname{Arcsec}$

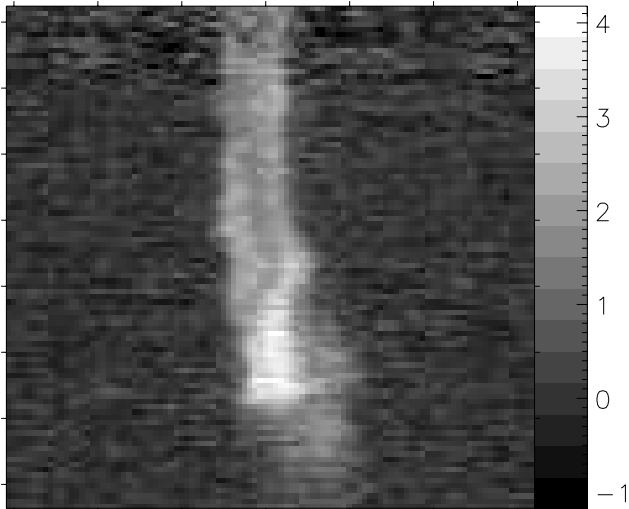

f) $\delta \mathrm{RA}=-416 \operatorname{Arcsec}$

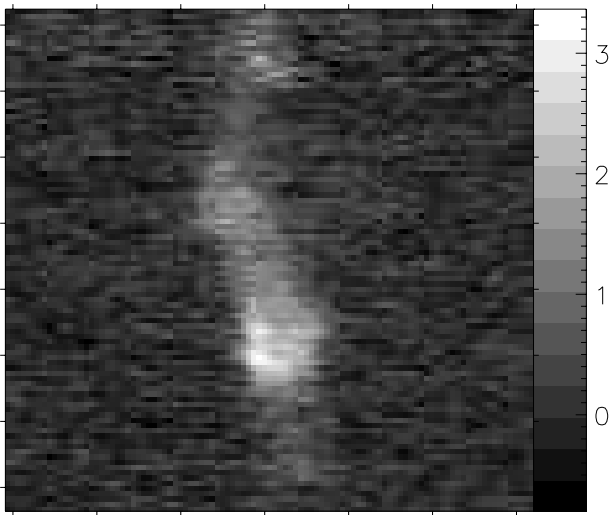

FIG. 3.-Declination-velocity diagrams for a set of cuts at different R.A. offsets, indicated above each diagram. These cuts, like those of Fig. 4, have been selected to be illustrative of the various morphologies of the velocity field in the map.

are uniform in space over a few tenths of a parsec. Table 1 lists values of the velocity gradients measured in the vicinity of the dense core. The dense core is localized within $\pm 100^{\prime \prime}$ in R.A. and from $+80^{\prime \prime}$ to $280^{\prime \prime}$ in declination. The six cuts listed in Table 1 intercept the dense core and have been selected because they illustrate the variations of the velocity gradients around the dense core. These variations are difficult to interpret because of projection effects. Yet it is unlikely that they trace a random velocity pattern around the dense core, because all the velocity components connect to the dense core position (in projection) at the dense core velocity. If these gradients trace inward motions toward the dense core, the variations of the sign of the gradients, on the same side of the core and from one side to the other, suggest that the inward flow is far from homogeneous and probably occurs along multiple and distinct directions around the 
a) $\delta \mathrm{Dec}=128 \mathrm{Arcsec}$

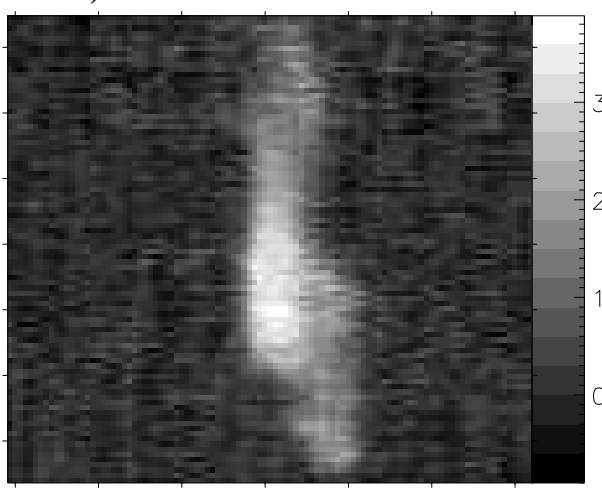

c) $\delta \mathrm{Dec}=352 \mathrm{Arcsec}$

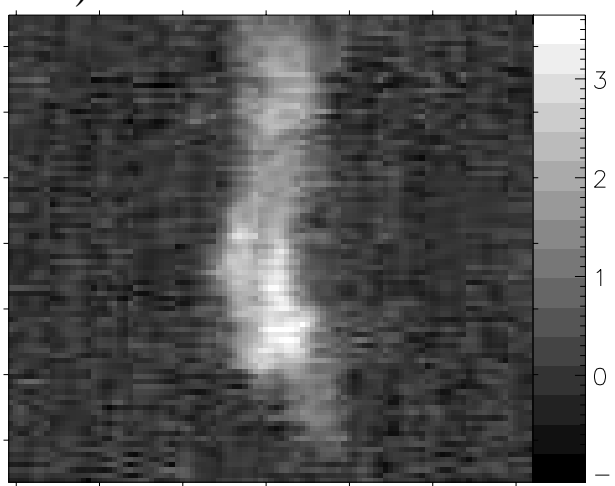

e) $\delta \mathrm{Dec}=640 \mathrm{Arcsec}$

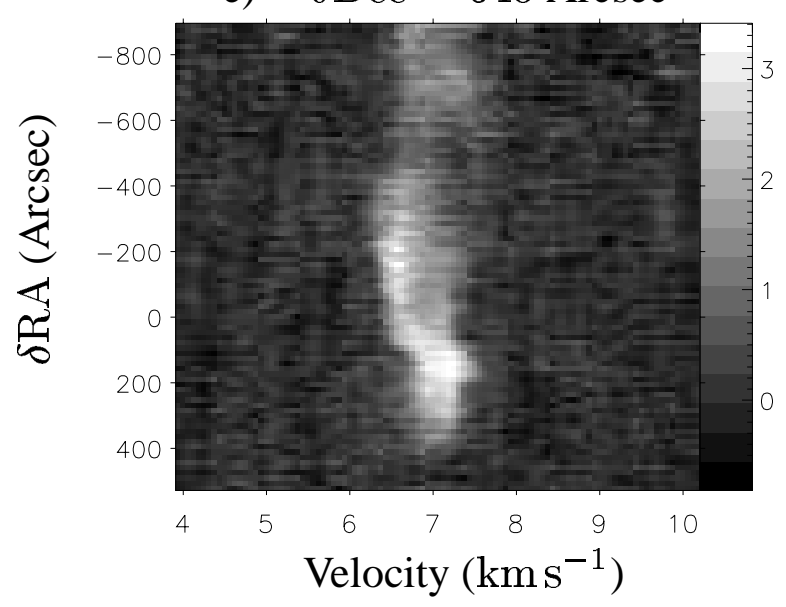

b) $\quad \delta \mathrm{Dec}=272 \operatorname{Arcsec}$

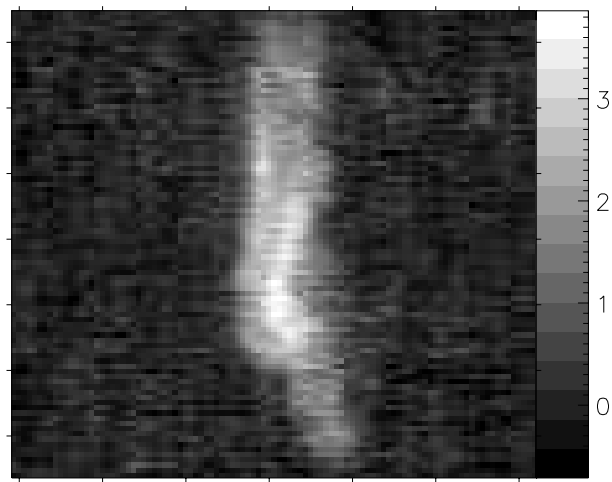

d) $\quad \delta \mathrm{Dec}=576 \operatorname{Arcsec}$

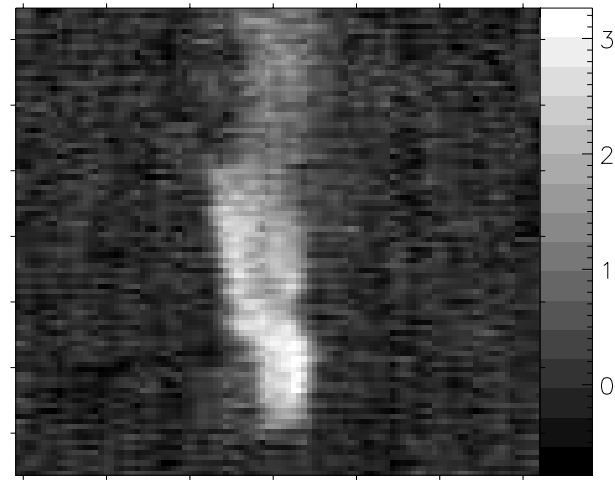

f) $\delta \mathrm{Dec}=784 \mathrm{Arcsec}$

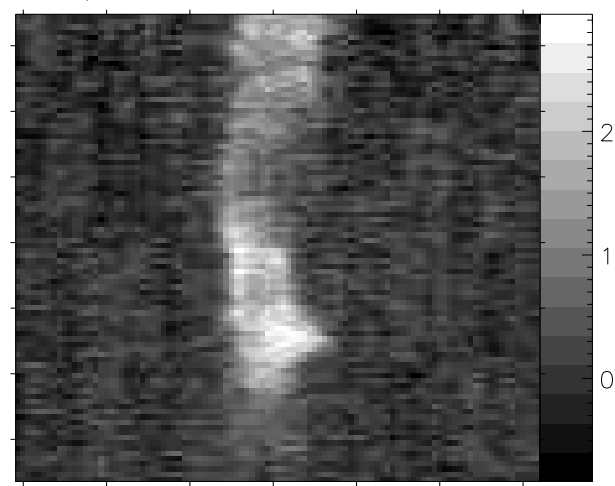

FIG. 4.-Same as Fig. 3, but for R.A.-velocity diagrams and cuts at different declination offsets

core. We rule out outward motions because the core has no embedded object. The maximum velocities of the gas relative to the core velocity are given in columns (4) and (7) of Table 1. They correspond to the product of the measured $\nabla v$ by its spatial extent. These relative velocities are large, up to $0.6 \mathrm{~km} \mathrm{~s}^{-1}$ at about $0.4 \mathrm{pc}$ from the core, and therefore larger than the sound velocity in $\mathrm{H}_{2}$ at $20 \mathrm{~K}\left(c_{s}=0.3\right.$ $\mathrm{km} \mathrm{s}^{-1}$ ), but they are commensurate with the Alfvén velocity, $v_{\mathrm{A}}=0.6 \mathrm{~km} \mathrm{~s}^{-1}$, for $B=10 \mu \mathrm{G}$ and $n_{\mathrm{H}_{2}}=500 \mathrm{~cm}^{-3}$.

Relatively large transverse gradients $\left(\sim 5 \mathrm{~km} \mathrm{~s}^{-1} \mathrm{pc}^{-1}\right)$ are visible across each of the two main filaments (1 and 4). Perhaps the most interesting feature is the fact that these gradients change sign along filament 1 , as illustrated in Figure 4. The filament emission covers the R.A. offset range $\left[0^{\prime \prime}, 200^{\prime \prime}\right]$ with slight changes with declination offsets (see Figs. 1 and 2). The velocity gradient across the filament is positive in Figure $4 c$ and negative in Figures $4 d$ and $4 e$. Gradient variations are also found over the western part of filament 4. A blow-up of the space-velocity diagrams is given in Figure $5 a$ for filament 4 and in Figure $5 b$ for filament 1 . The diagrams are restricted to the positions of the filaments. Velocity gradients measured at these positions for the line peak emission are plotted in Figure 6 as a function of the position along each filament. Fewer positions 
TABLE 1

Velocity Gradients in the Vicinity of the Dense CoRe

\begin{tabular}{lcccccc}
\hline \hline $\begin{array}{c}\text { Cut Position } \\
(1)\end{array}$ & $\begin{array}{c}\nabla v \text { South/East } \\
\left(\mathrm{km} \mathrm{s}^{-1} \mathrm{pc}^{-1}\right)\end{array}$ & $\begin{array}{c}\text { Extent } l \\
(\mathrm{pc}) \\
(3)\end{array}$ & $\begin{array}{c}\Delta v_{\max } \\
\left(\mathrm{km} \mathrm{s}^{-1}\right) \\
(4)\end{array}$ & $\begin{array}{c}\nabla v \text { North-West } \\
\left(\mathrm{km} \mathrm{s}^{-1} \mathrm{pc}^{-1}\right)\end{array}$ & $\begin{array}{c}\text { Extent } l \\
(\mathrm{pc}) \\
(6)\end{array}$ & $\begin{array}{c}\Delta v_{\max } \\
\left(\mathrm{km} \mathrm{s}^{-1}\right) \\
(7)\end{array}$ \\
\hline$\delta$ R.A. $=176^{\prime \prime} \ldots \ldots$. & -2.5 & 0.2 & 0.5 & 0.65 & 0.3 & 0.2 \\
$\delta$ R.A. $=80^{\prime \prime} \ldots \ldots$. & 0 & $\ldots$ & 0 & -1.2 & 0.3 & 0.36 \\
$\delta$ R.A. $=32^{\prime \prime} \ldots \ldots \ldots$ & 0 & $\ldots$ & 0 & 1.8 & 0.2 & 0.36 \\
$\delta$ R.A. $=-32^{\prime \prime} \ldots \ldots$ & -1 & 0.2 & 0.2 & 2.5 & 0.2 & 0.5 \\
$\delta$ decl. $=128^{\prime \prime} \ldots \ldots$. & -2.5 & 0.2 & 0.5 & 0 & $\ldots$ & 0 \\
$\delta$ decl. $=272^{\prime \prime} \ldots \ldots$. & -1.3 & 0.3 & 0.4 & 1.5 & 0.4 & 0.6 \\
\hline
\end{tabular}

NoTES.-For the cuts in declination (different $\delta$ R.A.), cols. (2)-(4) and cols. (5)-(7) characterize the velocity gradients measured in the south $(\mathrm{S})$ and north $(\mathrm{N})$ of the dense core, respectively. For the cuts in R.A. (different $\delta$ decl.), the same columns characterize the gradients measured in the east (E) and west (W) of the dense core, respectively. Note that $\Delta v_{\max }=l \nabla v$.
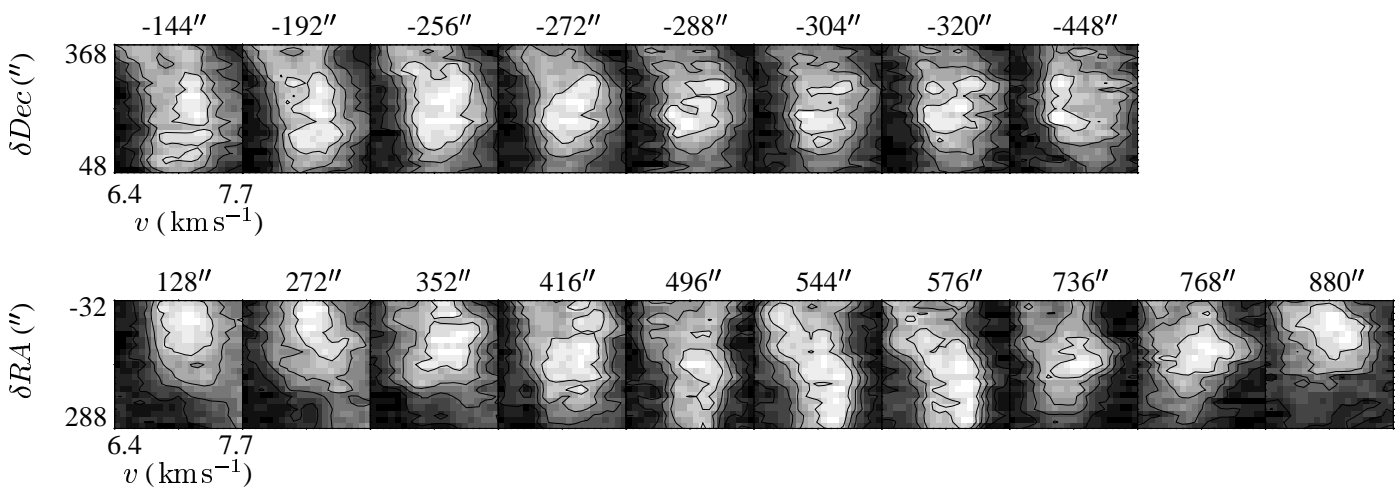

Fig. 5.-Blow-up of space-velocity diagrams over restricted ranges of space and velocity $\left(320^{\prime \prime}\right.$, or $0.24 \mathrm{pc}$, and $\left.1.3 \mathrm{~km} \mathrm{~s} \mathrm{~s}^{-1}\right)$. A velocity gradient of $1.3 \mathrm{~km}$ $\mathrm{s}^{-1}$ over $0.24 \mathrm{pc}$, or $5.5 \mathrm{~km} \mathrm{~s}^{-1} \mathrm{pc}^{-1}$ is therefore parallel to one of the diagonals of each square. The upper row displays declination cuts at different R.A. offsets (above each map) across filament 4. The filament extends from $\delta$ decl. $=144^{\prime \prime}$ to 304". The lower row displays R.A. cuts at different declinations across filament 1. The position of the filament varies in declination (see its shape in Fig. 1). It extends from $\delta$ R.A. $=200^{\prime \prime}$ to $0^{\prime \prime}$ in the south, between $\delta$ R.A. $=230^{\prime \prime}$ and $50^{\prime \prime}$ at about $\delta$ decl. $=550^{\prime \prime}$, and between $\delta$ R.A. $=150^{\prime \prime}$ and $-30^{\prime \prime}$ in the north.

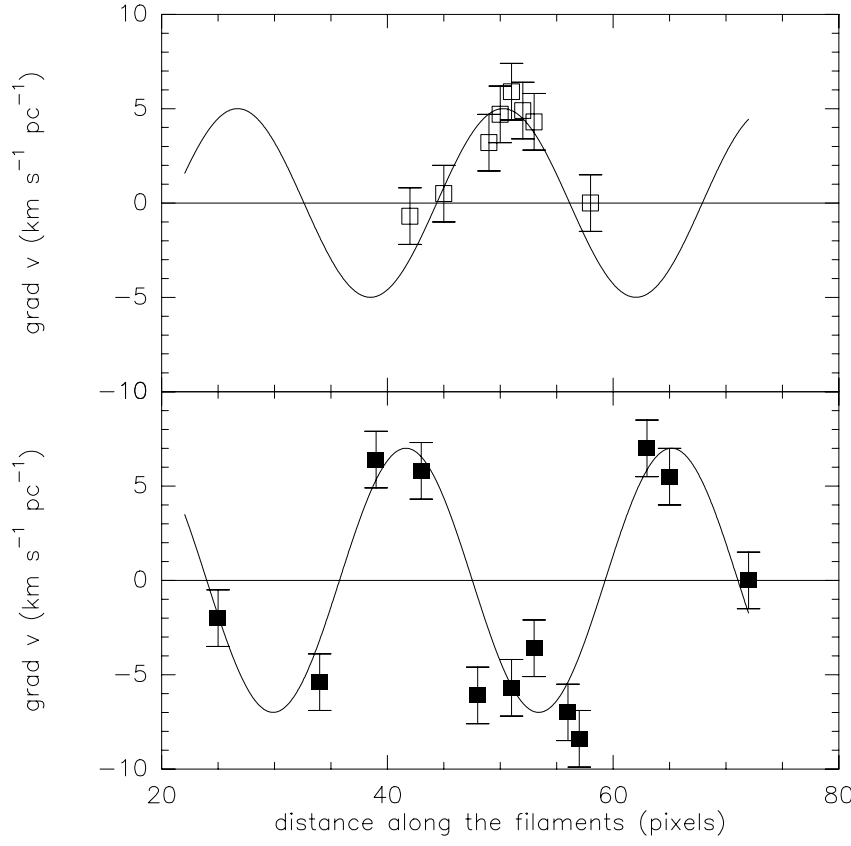

FIG. 6.-Variations of the transverse velocity gradients with position (expressed by the pixel number in each coordinate of the map; each pixel is $16^{\prime \prime}$, with origin in the southeast corner) along filaments 1 ( filled squares) and 4 (open squares). The sine curves are not fits to the data points; they illustrate the plausible similarity of the wavelength for both filaments. are available for filament 4 than for filament 1 because the linesareweakerand the velocityfieldismorecomplex.Thesame distance separates nulls and two successive maxima of velocity gradients in filament 1 . The corresponding wavelength for the variations is 23 pixels, or $0.28 \mathrm{pc}$. In filament 4 , two contiguous nulls are separated by 15 pixels, hence the wavelength for the gradient variations is $0.36 \mathrm{pc}$. It is remarkable that the wavelengths for the gradient variations, as well as the values of the maximum gradients, are so similar in both filaments. A tentative interpretation of these very conspicuous features is given in $\S 4$. These transverse gradients could be interpreted as signatures of rotation of the filaments, about axis-parallel to the filaments. Because of projection effects, the velocity gradient provides an upper limit to the period of the rotation according to $\tau_{\max }=6.3$ $\operatorname{Myr} / \nabla v$, where $\nabla v$ is expressed in $\mathrm{km} \mathrm{s}^{-1} \mathrm{pc}^{-1}$. For the two main filaments, this upper limit is therefore $\sim 1 \mathrm{Myr}$.

The velocity gradients and the velocity field at the parsec scale around the dense core are therefore characterized by three properties: (1) small $\left(\sim 1 \mathrm{~km} \mathrm{~s}^{-1} \mathrm{pc}^{-1}\right)$ and almost uniform longitudinal velocity gradients along the two main filaments; (2) larger velocity gradients within a few tenths of a parsec from the dense core, likely ascribed to inward motions with complex geometry and relative velocity closer to the Alfvén velocity than to the sound velocity; and (3) large transverse velocity gradients across the main 
filaments, ascribed to rotation, with spatial variations over $\sim 0.3 \mathrm{pc}$ along the filaments.

\subsection{Gas Density and Temperature}

Long integrations have been performed in the highfrequency lines ${ }^{12} \mathrm{CO}(J=4-3)$ and $(J=3-2)$ at three different offsets relative to the central position of the F98 maps: $\left(0^{\prime \prime},-15^{\prime \prime}\right),\left(0^{\prime \prime},-90^{\prime \prime}\right)$, and $\left(75^{\prime \prime}, 0^{\prime \prime}\right)$. The ${ }^{12} \mathrm{CO}(J=4-3)$ lines were observed in 1997 and 1998 as a check on the absolute line calibration. The difference between the two years was less than $20 \%$. These three positions were selected because they sample the line wing emission. The profiles are very similar at the three positions, and the lines have been averaged together. The resulting spectra are displayed in Figure 7.

Gaussian fits have been performed with three components for each spectrum. The two low-velocity components probably do not correspond to physically different

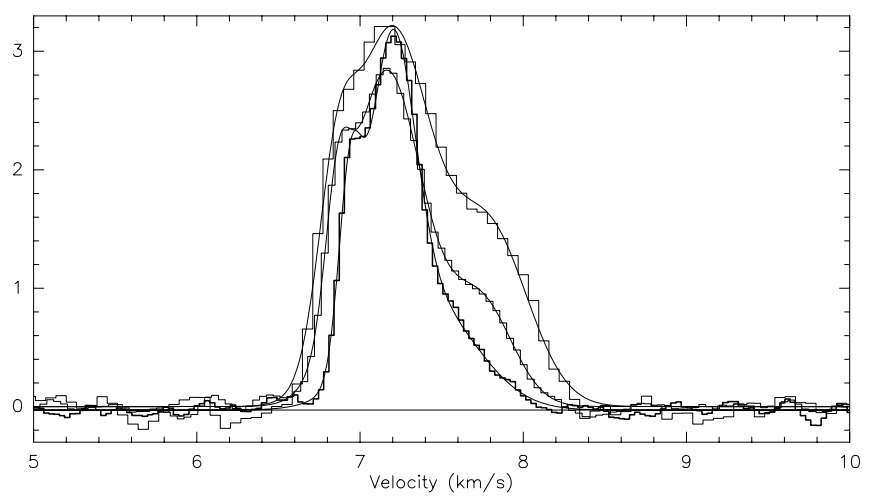

Fig. 7.- ${ }^{12} \mathrm{CO}(J=2-1)$ (thin solid line), $(J=3-2)$ (solid line), and $(J=4-3)$ (bold solid line) profiles obtained by integrating together the emission observed at offsets $\left(0^{\prime \prime},-15^{\prime \prime}\right),\left(0^{\prime \prime},-90^{\prime \prime}\right)$, and $\left(75^{\prime \prime}, 0^{\prime \prime}\right)$. The curves are the Gaussian fits described in the text. The temperature scale is the corrected main beam temperature of the ${ }^{12} \mathrm{CO}(J=2-1)$ line (F98). The ${ }^{12} \mathrm{CO}(J=4-3)$ line has been multiplied by 1.7 for clarity.

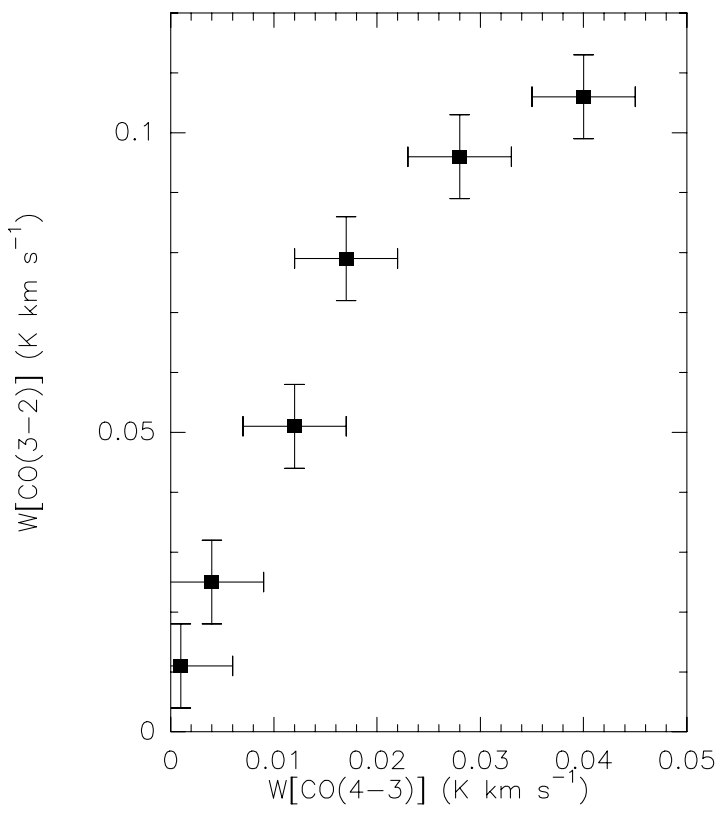

components because they are centered at the dense core velocity and are likely to be a single self-absorbed component. In each spectrum there is a third, higher-velocity component centered at $v_{\mathrm{lsr}}=7.8$ and $7.4 \mathrm{~km} \mathrm{~s}^{-1}$ for the $(J=3-2)$ and $(J=4-3)$ lines, respectively, with half-power widths $\Delta v=0.4$ and $0.8 \mathrm{~km} \mathrm{~s}^{-1}$, respectively. These values show that the high-velocity emission does not have the same distribution in each transition. The ${ }^{12} \mathrm{CO}(J=1-0)$ and $(J=2-1)$ line wing emission is still broader. Yet there is a velocity interval, $[7.8,8.2] \mathrm{km} \mathrm{s}^{-1}$, over which the intensities in each line stay proportional to each other (see Fig. 8 ), and it is on data from these channels that we build the argument below, because they are likely not to be contaminated by opacity effects.

The characteristics of the emission in the four lowest ${ }^{12} \mathrm{CO}$ transitions in this velocity interval are reported in Table 2. The temperature scale used for the two lowest transitions, $T_{\mathrm{mb}, c}$ is that introduced in F98. It is the mainbeam intensity corrected for the power collected in the secondary- and error-beam patterns at IRAM $30 \mathrm{~m}$. The higher- $J$ line ratios in extreme channels are roughly constant at $R(4-3 / 3-2)=0.22 \pm 0.05$. If ratios of efficiencies measured on small planets are taken into account (resolved emission), $R(4-3 / 3-2)=0.29 \pm 0.06$. We adopt $R(4-3 / 3-$ 2) $=0.25 \pm 0.1$ to accommodate the fact that the gas emitting in these transitions is neither in an unresolved structure nor very extended (see $\S 2$ ). We know from the low- $J$ observations (F98) that the ${ }^{12} \mathrm{CO}(J=2-1)$ line wing emission is not thermalized. The value $R(2-1 / 1-0)=0.65 \pm 0.15$ that we determined in F98 is significantly lower than the LTE values at kinetic temperatures consistent with the line brightness $\left(T_{k}>12 \mathrm{~K}\right)$. We therefore compute the populations of the $\mathrm{CO}$ rotational levels in the Large Velocity Gradient (LVG) approximation. To do so, we consider 33 levels, using the collision cross sections at large temperatures computed by McKee et al. (1982), implemented in an LVG radiative transfer code provided by M. Pérault.

To estimate the density and temperature of the line wing material, we look for solutions in the $\left[n_{\mathrm{H}_{2}}, N(\mathrm{CO}) / \Delta v\right]$

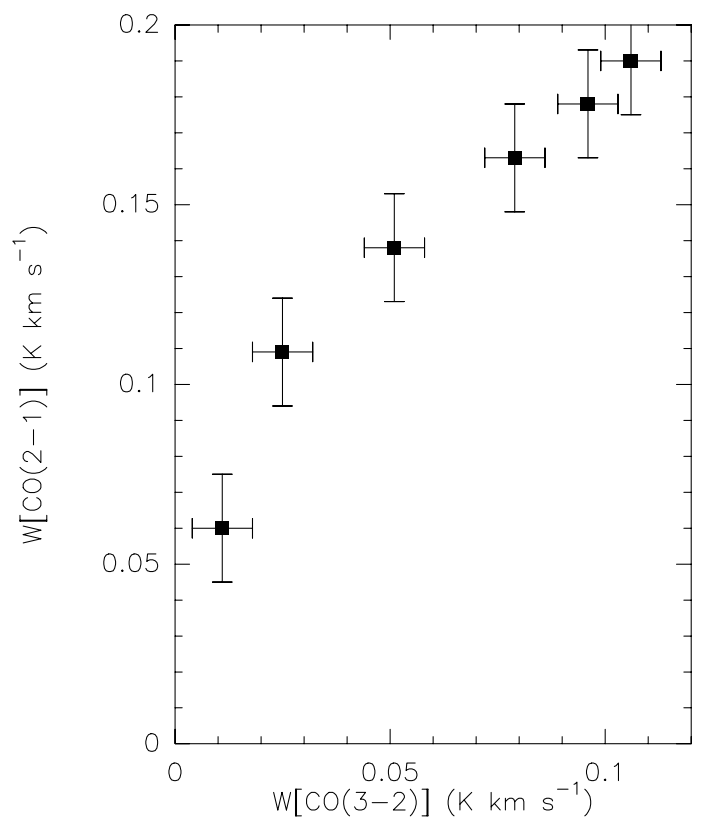

FIG. 8.-Left: ${ }^{12} \mathrm{CO}(J=3-2)$ vs. ${ }^{12} \mathrm{CO}(J=4-3)$ line intensities. Right: ${ }^{12} \mathrm{CO}(J=2-1)$ vs. ${ }^{12} \mathrm{CO}(J=3-2)$ line intensities over the extreme line wings. The values are line intensities integrated over velocity bins of $0.1 \mathrm{~km} \mathrm{~s}^{-1}$. 
TABLE 2

LINE INTENSITIES AND RATIOS

\begin{tabular}{ccccccc}
\hline \hline $\begin{array}{c}T_{\mathrm{mb}, c}(1-0) \\
(\mathrm{K})\end{array}$ & $\begin{array}{c}T_{\mathrm{mb}, c}(2-1) \\
(\mathrm{K})\end{array}$ & $\begin{array}{c}T_{\mathrm{mb}}(3-2) \\
(\mathrm{K})\end{array}$ & $\begin{array}{c}T_{\mathrm{mb}}(4-3) \\
(\mathrm{K})\end{array}$ & $R(2-1 / 1-0)$ & $R(3-2 / 2-1)$ & $R(4-3 / 3-2)$ \\
\hline 2.5 & 1.5 & $0.9(0.03)$ & $0.25(0.03)$ & $0.65 \pm 0.15$ & $0.6 \pm 0.1$ & $0.25 \pm 0.1$ \\
\hline
\end{tabular}

Notes.-Line intensities are at $7.8 \mathrm{~km} \mathrm{~s}^{-1}$, and line ratios are in the extreme line wings of the emission, integrated over the three positions observed at high frequency. The numbers in parentheses are the rms noise levels of the high-frequency spectra.

plane that satisfy $R(4-3 / 3-2)=0.25 \pm 0.1$ and $R(2-1 /$ $1-0)=0.65 \pm 0.15$ and have a ${ }^{12} \mathrm{CO}(J=4-3)$ line intensity greater than or equal to that observed, i.e., $f_{s}=$ $T_{\mathrm{CO}}(4-3)_{\mathrm{obs}} / T_{\mathrm{CO}}(4-3)_{\mathrm{LVG}} \leq 1$, to allow for beam dilution. Sets of solutions are given in Table 3 for a range of temperatures. For each pair $\left[n_{\mathrm{H}_{2}}, N(\mathrm{CO}) / \Delta v\right]$ satisfying the above three observational constraints, we compute the $\mathrm{CO}$ abundance inferred from the wing velocity coverage $\Delta v=0.8 \mathrm{~km} \mathrm{~s}^{-1}$, where the depth of the line of sight is taken to be equal to the thickness of the filaments, $L=0.1$ pc, because the line wing emission originates primarily in the filaments, i.e.,

$$
X(\mathrm{CO})=\frac{N(\mathrm{CO})}{\Delta v} \frac{\Delta v}{n_{\mathrm{H}_{2}} L} .
$$

Therefore, solutions that would imply $X(\mathrm{CO})>8 \times 10^{-5}$ or $f_{s}>1$ are ruled out. Table 3 shows that there is no solution for $T_{k}<20 \mathrm{~K}$ or for $T_{k}>250 \mathrm{~K}$. But a whole range of solutions exists for $20 \mathrm{~K} \leq T_{k} \leq 250 \mathrm{~K}$ : solutions $4,6,7,10$, and 13 of Table 3 and a solution with $n_{\mathrm{H}_{2}}=180 \mathrm{~cm}^{-3}$ at $T_{k}=250 \mathrm{~K}$. Note that an upper limit to the gas temperature is provided by the width of the line wing emission. If the $\mathrm{CO}$ motions are thermal, the half-power line width in the ${ }^{12} \mathrm{CO}$ $(J=4-3)$ line of $0.8 \mathrm{~km} \mathrm{~s}^{-1}$ corresponds to an upper limit of $375 \mathrm{~K}$.

The thermal pressure of each solution is given in the last column of Table 3. These thermal pressures are all larger than the confining pressure provided by the weight of the
$\mathrm{H}$ I layer in the Galaxy $\left(P_{\mathrm{H}} / k=10^{4} \mathrm{~K} \mathrm{~cm}^{-3}\right.$ at midplane; Boulares \& Cox 1990). We estimate that this pressure, at the distance of the Taurus molecular complex from midplane $(z=50 \mathrm{pc})$, is reduced by a factor of 2 and therefore that the ambient pressure is only $P_{\mathrm{Hi}} / k \sim 5 \times 10^{3} \mathrm{~K} \mathrm{~cm}^{-3}$. Nonthermal motions increase the internal pressure within the filaments according to $P_{i, \mathrm{nt}}=\rho_{i} \bar{v}_{i, \mathrm{nt}}^{2} / 3$, with $\bar{v}_{i, \mathrm{nt}}=1.6 \sigma_{i, \mathrm{nt}}$. The nonthermal contribution to the observed line width, $\Delta v=0.8 \mathrm{~km} \mathrm{~s}^{-1}$, is obtained by deconvolution from the thermal contribution. In the same units, the nonthermal pressure within the filaments is found to vary from $P_{i, \mathrm{nt}} / k \sim$ $3 \times 10^{4}$ to $10^{3} \mathrm{~K} \mathrm{~cm}^{-3}$ for all the solutions between $T_{k}=$ $20 \mathrm{~K}$ and $250 \mathrm{~K}$. It is therefore not the dominant contribution to the internal pressure within the filaments, although it is not negligible, in particular at low temperature, where the thermal and nonthermal contributions are similar. Nonthermal motions in the gas outside the filaments contribute to the confinement of the filaments. This contribution is estimated as follows. Because $75 \%$ of the mass traced by ${ }^{12} \mathrm{CO}(J=2-1)$ in the map lies in the filaments and because the fractional areas of the filaments and background in the map are the same, the average column density across the filaments is about 3 times as high as that of the background. The average density within the filaments, $\rho_{i}$, is therefore related to that of the environment, $\rho_{\text {out }}$, through the ratio of the path lengths across the filaments and the surrounding gas, $\rho_{i}=3 \rho_{\text {out }} L_{\text {out }} / L_{\text {fil }} \approx 15 \rho_{\text {out }}$ for $L_{\text {out }}=1 \mathrm{pc}$ (Ungerechts \& Thaddeus 1987) and $L_{\mathrm{fil}}=0.2 \mathrm{pc}$, an

TABLE 3

LVG SoLuTions

\begin{tabular}{|c|c|c|c|c|c|c|}
\hline Solution & $\begin{array}{c}T_{k} \\
(\mathrm{~K})\end{array}$ & $\begin{array}{c}n_{\mathrm{H}_{2}} \\
\left(\mathrm{~cm}^{-3}\right)\end{array}$ & $\begin{array}{c}N(\mathrm{CO}) / \Delta v \\
\left(\mathrm{~cm}^{-2} / \mathrm{km} \mathrm{s}^{-1}\right)\end{array}$ & $X(\mathrm{CO})$ & $f_{s}$ & $\begin{array}{c}P_{\mathrm{th}} / k \\
\left(\mathrm{~K} \mathrm{~cm}^{-3}\right)\end{array}$ \\
\hline 1. & 10 & $10^{3}$ & $10^{17}$ & $2.8 \times 10^{-4}$ & 1.2 & $10^{4}$ \\
\hline $2 \ldots \ldots \ldots$ & 10 & $10^{4}$ & $3 \times 10^{16}$ & $8 \times 10^{-6}$ & 2.36 & $10^{5}$ \\
\hline $3 \ldots \ldots \ldots$ & 20 & $10^{3}$ & $3 \times 10^{16}$ & $8 \times 10^{-5}$ & 0.36 & $2 \times 10^{4}$ \\
\hline $4 \ldots \ldots \ldots$ & 20 & $2 \times 10^{3}$ & $10^{16}$ & $1.4 \times 10^{-5}$ & 0.5 & $4 \times 10^{4}$ \\
\hline $5 \ldots \ldots$ & 20 & $3 \times 10^{3}$ & $5 \times 10^{15}$ & $4.8 \times 10^{-6}$ & 0.6 & $6 \times 10^{4}$ \\
\hline $6 \ldots \ldots$ & 40 & 600 & $10^{16}$ & $4.4 \times 10^{-5}$ & 0.6 & $2.4 \times 10^{4}$ \\
\hline $7 \ldots \ldots$ & 40 & 650 & $5 \times 10^{15}$ & $2.1 \times 10^{-5}$ & 1 & $2.6 \times 10^{4}$ \\
\hline $8 \ldots \ldots$ & 40 & $10^{3}$ & $2 \times 10^{15}$ & $5.6 \times 10^{-6}$ & 2 & $4 \times 10^{4}$ \\
\hline $9 \ldots \ldots$ & 60 & 300 & $2 \times 10^{16}$ & $1.9 \times 10^{-4}$ & 0.5 & $1.8 \times 10^{4}$ \\
\hline $10 \ldots \ldots$ & 60 & 400 & $5 \times 10^{15}$ & $3.5 \times 10^{-5}$ & 1 & $2.4 \times 10^{4}$ \\
\hline $11 \ldots \ldots$ & 60 & 600 & $10^{14}$ & $4.8 \times 10^{-7}$ & 4 & $3.6 \times 10^{4}$ \\
\hline $12 \ldots \ldots$ & 100 & 150 & $2 \times 10^{16}$ & $4.0 \times 10^{-4}$ & 0.5 & $1.5 \times 10^{4}$ \\
\hline $13 \ldots \ldots$ & 100 & 300 & $5 \times 10^{15}$ & $4.8 \times 10^{-5}$ & 1 & $3 \times 10^{4}$ \\
\hline $14 \ldots \ldots$ & 100 & 450 & $10^{14}$ & $6.4 \times 10^{-7}$ & 3 & $4.5 \times 10^{4}$ \\
\hline $15 \ldots \ldots$ & 250 & 100 & $10^{16}$ & $2.8 \times 10^{-4}$ & 0.71 & $2.5 \times 10^{4}$ \\
\hline $16 \ldots \ldots$ & 250 & 170 & $5 \times 10^{15}$ & $8 \times 10^{-5}$ & 0.83 & $4.2 \times 10^{4}$ \\
\hline $17 \ldots \ldots$ & 250 & 250 & $10^{15}$ & $1.1 \times 10^{-5}$ & 3 & $6.2 \times 10^{4}$ \\
\hline
\end{tabular}

Notes.-LVG solutions satisfying $R(4-3 / 3-2)=0.25 \pm 0.1$ and $R(2-1 / 1-0)=$ $0.65 \pm 0.15$ for a broad range of kinetic temperatures. Solutions $4,6,7,10$, and 13 are consistent with the additional constraints $f_{s} \leq 1$ and $X(\mathrm{CO})<8 \times 10^{-5}$. 
average value of the projected size of the filaments $\left(\approx 300^{\prime \prime}\right)$ at the emission level of $1 \mathrm{~K} \mathrm{~km} \mathrm{~s}^{-1}$. The line widths outside the filaments are at most 1.5 times as large as those within (see the space-velocity cuts of Figs. 3 and 4). Assuming that these line widths are dominated by a nonthermal contribution provides an upper limit to the turbulent pressure, $P_{\text {out }, \mathrm{nt}} / k=\rho_{\text {out }} \bar{v}_{\text {out }, \mathrm{nt}}^{2} / 3$, exerted by their environment on the filaments. For $\rho_{\text {out }}=\rho_{i} / 15$ and $\bar{v}_{\text {out,nt }}=$ $1.5 \bar{v}_{i, \mathrm{nt}}$, the nonthermal pressure exerted by the environment on the filaments is $P_{\text {out nt }}=0.15 P_{i, \text { nt }}$ and thus ranges between $4.5 \times 10^{3}$ and $165 \mathrm{~K} \mathrm{~cm}^{-3}$, depending on the solution adopted for the gas density within the filaments.

Therefore, the total internal pressure inside the filaments (thermal plus nonthermal) is found to range between $7 \times 10^{4}$ and $4.6 \times 10^{4} \mathrm{~K} \mathrm{~cm}^{-3}$, while the external pressure (weight of the $\mathrm{H}$ I layer plus external turbulent pressure) does not exceed $10^{4} \mathrm{~K} \mathrm{~cm}^{-3}$. This is insufficient to balance the internal pressure of the filaments. In $\S 4$, we discuss the issue of the confinement of the filaments.

The above set of solutions assumes a single gas component. Yet other solutions are possible, where very dense and cold cells, unresolved by existing observations, are coated by lower density gas of large optical depth in the low- $J$ transitions. In such two-component solutions, the $(J=1-0)$ emission is dominated by that of the low-density envelope of each cell, while the ${ }^{12} \mathrm{CO}(J=4-3)$ emission arises mostly in the densest and coldest parts. The ${ }^{12} \mathrm{CO}$ $(J=2-1)$ and $(J=3-2)$ emissions are a combination of both. The problem with such solutions lies in the fact that the envelope of each cell must be quite optically thick in the ${ }^{12} \mathrm{CO}(J=1-0)$ line. It is possible, though. For instance, the cells proposed in F98 to explain the low- $J$ CO line properties [sheetlike or filamentary cells of $200 \mathrm{AU}$, cold $\left(T_{k}<20\right.$ $\mathrm{K})$, with density $\left.n_{\mathrm{H}_{2}}=3 \times 10^{3} \mathrm{~cm}^{-3}\right]$ are optically thick in the ${ }^{12} \mathrm{CO}(J=1-0)$ and $(J=2-1)$ lines but would not be detected in the ${ }^{12} \mathrm{CO}(J=4-3)$ line at the level observed, even though they would have a surface filling factor of unity. Each cell would need to have a denser, much smaller core, responsible for the observed ${ }^{12} \mathrm{CO}(J=4-3)$ emission. Cores of density $n_{\mathrm{H}_{2}}=2 \times 10^{4} \mathrm{~cm}^{-3}$ or higher and $N(\mathrm{CO}) /$ $\Delta v=10^{16} \mathrm{~cm}^{-2}\left(\mathrm{~km} \mathrm{~s}^{-1}\right)^{-1}$ would be a plausible solution to explain the strength of the ${ }^{12} \mathrm{CO}(J=4-3)$ wing. Yet size scales as small as $40 \mathrm{AU}$ are inferred for these cores and the problem of beam dilution is raised, unless these cores too have nonspherical geometries and large surface filling factor as a whole.

In a recent study, Hegmann \& Kegel (2000) have shown that density fluctuations strongly affect the line profiles and line intensity ratios in a turbulent velocity field. They find, for instance, that the line intensity ratio $R(4-3 / 1-0)$ increases by a factor of 2 when the dispersion of the onepoint distribution function of the density is increased by a factor of 20, for the same turbulent velocity field and for the same correlation length as for the density. Of course, the two density distributions are very different: in the first case half the mass is contained in gas at densities higher than the average density, while in the latter case half the mass is in clumps of density more than 10 times the average density. Yet this example illustrates the high sensitivity of the line ratios, in particular those of well-separated rotational transitions, to the structures of the velocity and density fields (dispersions and correlation lengths). Interestingly, Ingalls et al. (2000) have reported observations of high-latitude clouds in the four lowest rotational transitions of $\mathrm{CO}$. They find that all the integrated line intensities are linearly correlated and infer, as Falgarone \& Phillips (1996) and Falgarone et al. (1998) did, that the excitation conditions are uniform in these clouds. From their line ratios, in particular the large intensities of the $\mathrm{CO}(J=4-3)$ lines, they conclude that the solution is high-density, low-temperature gas with CO-emitting cells $\sim 2000$ AU. Clearly, from our Figure 8, we cannot state that the different line emissions are linearly correlated across the line wings, and we conclude that the gas emitting in the line wings in the vicinity of L1512 has different physical properties than that dominating the emission of high-latitude molecular clouds.

A detailed model of individual cells, including geometry and velocity field, is warranted to further quantify a solution with large density (and temperature) gradients at small scale $(<200 \mathrm{AU})$. This must await new observations with sensitive interferometers in the millimeter line domain and theoretical developments regarding cell structure, because at present we are not able to sufficiently constrain the geometry and the velocity field of the CO-emitting cells at such small scales.

\section{DISCUSSION}

\subsection{The Plausibility of the Hot Diluted Solutions}

It is somewhat disappointing to find out that $\mathrm{CO}$ lines in four different rotational transitions and a large-scale map are not yet sufficient to determine unambiguously the density and temperature structure of the filaments surrounding a nearby dense core.

However, the set of low-density and warm solutions is consistent with recent detections of large abundances of $\mathrm{HCO}^{+}$in these filaments (Falgarone et al. 2001, in preparation). The large abundances inferred from the line intensity of the $\mathrm{HCO}^{+}(J=1-0)$ line detected in emission are inconsistent with steady-state chemistry in gas at density lower than $10^{4} \mathrm{~cm}^{-3}$. These large abundances can be explained by nonequilibrium chemistry triggered in hot regions, transiently heated by bursts of dissipation of turbulent energy. The high temperatures reached by the gas in the dissipation bursts allow endothermic reactions (or reactions with large energy barriers) to be activated. These bursts of dissipation are associated either with MHD shocks (Flower $\&$ Pineau des Forêts 1998) or with coherent, strong vortices bounded by layers of large velocity shear, in which viscous dissipation is large (Joulain et al. 1998). Subsequent isobaric cooling of these regions, once the burst has switched off, and the concomitant photodissociation of the molecules formed during the burst of dissipation have been computed. The $\mathrm{HCO}^{+}$abundances are consistent with those observed when the gas densities are between 50 and $10^{3} \mathrm{~cm}^{-3}$ with gas kinetic temperatures between $200 \mathrm{~K}$ and $50 \mathrm{~K}$, depending on the external shielding from the UV field. The time elapsed after the end of the burst, when the gas has cooled down to $200 \mathrm{~K}$ and $X\left(\mathrm{HCO}^{+}\right)=2 \times 10^{-9}$, for instance, is short, $2 \times 10^{3} \mathrm{yr}$ for an ambient visual extinction $A_{v}$ of 1 mag. At lower extinction, it would be even shorter.

Although we cannot rule out that these large $\mathrm{HCO}^{+}$ abundances originate in very dense, small, and cold cells such as those envisioned in the previous section, it is striking that nonequilibrium processes triggered by intermittent dissipation of turbulent energy are so consistent with the data. 


\subsection{The Confinement of the Filaments by a Helical Magnetic Field}

We turn now to the dynamics of the two main filaments. We estimate their mass per unit length to be $m=10^{15} \mathrm{~g}$ $\mathrm{cm}^{-1}=1.6 M_{\odot} \mathrm{pc}^{-1}$ for an average density $n_{\mathrm{H}_{2}}=10^{3}$ $\mathrm{cm}^{-3}$ within a radius of $0.1 \mathrm{pc}$, corresponding to the average half-power size. Their one-dimensional internal velocity dispersion $\left(\sigma=0.13 \mathrm{~km} \mathrm{~s}^{-1}\right)$ is derived from the $\mathrm{CO}$ half-power line width $\left(\Delta v=0.2 \mathrm{~km} \mathrm{~s}^{-1}\right)$. The virial mass per unit length is $m_{\mathrm{vir}}=2 \sigma^{2} / G=7.5 \mathrm{M}_{\odot} \mathrm{pc}^{-1}$, so that the filaments are far below virial balance between selfgravity and internal kinetic energy, $m / m_{\text {vir }}=0.2$. They are not held either by the pressure of the $\mathrm{H}_{\mathrm{I}}$ layer or by the turbulent pressure of their environment, as shown in $\S 3$. We therefore consider magnetic confinement.

In recent papers, Fiege \& Pudritz (2000a, 2000b) study the equilibria of pressure-truncated filaments held by selfgravity and/or helical magnetic fields. They find that observed properties of filaments in molecular clouds, namely $m / m_{\text {vir }}$ and $P_{s} / \bar{P}$, the ratio of the surface pressure to internal average pressure, are more consistent with models with helical fields than either unmagnetized models or those with purely poloidal fields. They stress that, for magnetic field intensities decreasing with the radius of the filaments, the poloidal component of the field, $B_{z}$, helps in supporting filaments against self-gravity and that the toroidal component, $B_{\phi}$, squeezes the filaments. Helical fields therefore either support or confine filaments, depending on the ratio $\Gamma_{\phi} / \Gamma_{z}$ of the toroidal and poloidal flux to mass ratios. They study the linear stability of these equilibrium configurations to axisymmetric perturbations. Their results appear to be particularly relevant to the present observations, as we explain below.

Fiege \& Pudritz (2000a, 2000b) find that the gravitydriven modes, which dominate when $\Gamma_{\phi}$ is weak compared to $\Gamma_{z}$, tend to generate long (compared to the thickness of the filaments) wavelength instabilities, whereas in the opposite condition, $\Gamma_{\phi}>\Gamma_{z}$, MHD modes develop with much smaller wavelengths. Gravity-driven and MHD modes blend together at intermediate wavelengths and correspond to the physical regime in which the filaments fragment most slowly. The striking point in their results is that these instabilities induce toroidal velocities in the filaments that change sign with a spatial period equal to the wavelength of the instability. This is a pattern extremely reminiscent of what has been observed in the two main filaments (Figs. 5 and 6).

We have therefore estimated from their computations which kind of instability the observed flipping of the transverse velocity gradients traces. It is determined by the ratio, $\lambda / D$, of the observed wavelength $(\lambda)$ to the filaments' diameter $(D \sim 0.1 \mathrm{pc})$, under the assumption that the observed wavelength is that of the mode of fastest-growing instability $\left(\lambda_{\max }\right)$ for a given value of $\Gamma_{\phi} / \Gamma_{z}$. The observed ratio is $\lambda / D \sim 3$ for the two main filaments discussed in $\S 3$. This corresponds to an MHD instability developing in a toroidally dominated magnetic field, and it is indeed very close to the intermediate regime in which self-gravity starts to be important and the growth rates of the instability are the smallest. The determination of the ratio $B_{\phi} / B_{z}$ requires knowledge of the dependence of the gas density on radius, which has not yet been determined observationally. According to Fiege \& Pudritz (2000b), the growth rate of this instability is $3 \times 10^{-15} \mathrm{~s}^{-1}<\omega<10^{-14} \mathrm{~s}^{-1}$, which corre- sponds to rather long timescales, between $5 \times 10^{5}$ and $1.6 \times 10^{6} \mathrm{yr}$.

In such a regime, the toroidal component $B_{\phi}$ has two roles: it helps in confining the filament and has a stabilizing influence. The variation with radius of the toroidal component induces a $z$ component of the current $j=\nabla \times B$, hence a radial component of the Lorentz force. The intensity of the Lorentz force may be approximated by $F_{L} \approx$ $B^{2} / 4 \pi r_{0}$, where $B$ is the intensity of the field and $r_{0}$ the thickness of the filament, over which $B_{\phi}$ significantly changes. For $B=10 \mu \mathrm{G}$ and $r_{0}=0.1 \mathrm{pc}, F_{L} \sim 2 \times 10^{-29}$ dynes $\mathrm{cm}^{-3}$. The radial component of the Lorentz force helps in confining the filament because the drag force between the ions and neutrals has a comparable amplitude. The force per unit volume exerted by the ions on the neutrals via collisions is $F_{i n}=\gamma \rho_{i} \rho_{n}\left(v_{i}-v_{n}\right)$ where $\gamma=$ $\langle\sigma v\rangle_{i n} /\left(\mu_{i}+\mu_{n}\right) \sim 0^{14} \mathrm{~cm}^{3} \mathrm{~s}^{-1} \mathrm{~g}^{-1}$ (main ion $\mathrm{HCO}^{+}$), $\rho_{i}$ and $\rho_{n}$ are the mass densities of ions and neutrals, and $v_{i}-v_{n}$ is the amplitude of the drift velocity. It is interesting that $F_{\text {in }}=F_{L}$ for $n_{\mathrm{H}_{2}}=500 \mathrm{~cm}^{-3}, v_{i}-v_{n}=0.3 \mathrm{~km} \mathrm{~s}^{-1}$, and an ionization degree of $x=2 \times 10^{-6}$. Were the magnetic field structured at smaller scales without being more intense, the Lorentz force would increase because the current vector would increase, but the confinement of the neutrals by the field would be efficient only if the drag force between ions and neutrals increases accordingly, i.e., for larger densities or larger drift velocities.

Confinement of dense cores with poloidal fields requires that the magnetic fields rise toward the edges of the cores, squashing them into a prolate shape (J. D. Fiege 2000, private communication). Tagger, Falgarone, \& Shukurov (1995) and, more recently, Franqueira, Tagger, \& Gómez de Castro (2000) have shown that, if neutrals and magnetic field decouple at small scale, ambipolar filamentation of the magnetic field under the effect of turbulence causes the ions and magnetic flux to condense into structures from which the neutrals are progressively expelled as time increases. In these regions, the neutrals compress the ions and the field and reciprocally, the ions and field compress the neutrals.

This tentative interpretation of our observations, namely the flipping of the transverse velocity gradients along the filaments, provides considerable evidence for helical field in a filamentary molecular structure. The ratio of the wavelength of the instability to the filament thickness is the first dynamical manifestation that the toroidal component is the dominant component of the magnetic field. This toroidal component has the important role of confining the filaments, which are far from being held by self-gravity $\left(\mathrm{m} / \mathrm{m}_{\mathrm{vir}}=0.2\right)$, by pressure of the H I component, or by turbulent pressure.

\subsection{Slow Gas Accretion onto the Dense Core}

The longitudinal velocity gradients observed along the filaments and within a few tenths of a parsec from the core may trace gas motions toward the dense core. It is not possible, without a detailed model of the core, to determine what drives the inward motion, in particular if it is due to a nonthermal pressure gradient between the core and its environment. Gravity has to play a role, but magnetic forces, as discussed in the previous paragraph, are expected to strongly modify the collapse.

We can estimate the accretion rate of the environment onto the dense core from the velocity gradients observed in the dense core environment (Table 1). If we assume that 
accretion is taking place in $4 \pi / 8$ steradians (as suggested by the geometry in projection) and that the average density of the infalling gas is $n_{\mathrm{H}_{2}}=500 \mathrm{~cm}^{-3}$, we find that the accretion rate is of the order of $\dot{M}=4 \times 10^{-6} M_{\odot} \mathrm{yr}^{-1}$ $(r / 0.2 \mathrm{pc})^{2}\left(v_{\text {rel }} / 0.5 \mathrm{~km} \mathrm{~s}^{-1}\right)$. This is an upper limit, however, since the infalling gas may not fill $4 \pi / 8$ steradians and may be structured over smaller solid angles, as suggested by the spatial variations of the gradients in the core vicinity. A range of relative velocities of accreting gas relative to the dense core is provided by the values of $v_{\text {rel }}$ in Table 1 , and $r$ is the distance from the core center at which this velocity is measured.

L1512 therefore appears to be a very young dense core which is likely to be still growing by gas accretion. This would be consistent with the fact that L1512 is one of the least centrally condensed of the nearby dense cores mapped in the submillimeter continuum emission (Ward-Thompson et al. 1994) and has no signpost of infall motions toward a central object, such as the so-called blue-line asymmetry (Gregersen \& Evans 2000).

It may be helpful here to stress that the inward motions observed in the vicinity of $\mathrm{L} 1512$ are of a different nature from those detected within $\sim 0.03 \mathrm{pc}$ of the center of several dense cores, interpreted as possible infall motions related to star formation (Lee, Myers, \& Tafalla 1999). They may also differ from the so-called extended inward motions detected in L1544 by Tafalla et al. (1998): those inward motions extend over at most $0.1 \mathrm{pc}$ from the center of the core and are identified with optically thin tracers, while they are here observed up to almost $1 \mathrm{pc}$ from the core in L1512. The size scales over which these inward motions are observed therefore differ by almost 1 order of magnitude. In addition, the relative velocities between the infalling gas and the core are larger at large scale in L1512 than at small scale in L1544. Yet these motions might coexist in both cores: the L1544 maps of Tafalla et al. (1998) do not extend far enough from the core, and our analysis based on ${ }^{12} \mathrm{CO}$ cannot probe the inner $0.1 \mathrm{pc}$ of L1512. In L1512, the puzzle raised by the fact that the relative velocity increases with the distance from the core is left unsolved, except for the possibility of the gravitational collapse of a mass of gas at uniform density in a very early phase (Spitzer 1978).

\section{CONCLUSIONS}

The parsec-scale environment of the starless dense core
L1512 is characterized by conspicuous filaments, which contain the bulk of the mass (as traced by ${ }^{12} \mathrm{CO}$ ) and converge in projection and in velocity on the dense core location. The gas in this environment reveals a high level of complexity.

First, the gas is far from quiescent, and various motions are observed at different scales. There is spinning of the filaments along their long axis, with velocity gradients up to $\sim 8 \mathrm{~km} \mathrm{~s}^{-1} \mathrm{pc}^{-1}$ implying a rotation period $\sim 1$ Myr. There are inflow motions toward the dense core along the main filaments (up to about $1 \mathrm{pc}$ from the core), but also along more distributed directions, over a few tenths of parsec from the core, at velocities (relative to that of the dense core) likely to be closer to the Alfvén velocity than to the sound velocity. The gas accretion rate to the core is low, very likely mediated and slowed by strong magnetic forces.

Second, we find oscillations in the toroidal velocity of the filaments, and a tentative interpretation is proposed: it may be the signature of an MHD instability developing in filaments threaded by a helical magnetic field. In the present case of filaments not confined by self-gravity nor by external pressure, the toroidal component dominates the field and contributes to the confinement of the gas.

Last, there is evidence for the existence of warm and diluted gas in the environment of this dense core. This gas is at present out of chemical and thermal balance and may have been formerly heated by a burst of viscous dissipation of turbulence, switched off a few thousand years ago.

These observational properties suggest that rotation seems to manifest itself more than compression in the environment of this dense core and that the magnetic field plays a major role in mediating the gas infall leading to the growth of the dense core.

One of us (E. F.) is grateful to R. Pudritz and J. Fiege for illuminating confrontations between observations and theory, which have inspired the tentative identification of the MHD instability in the filaments. E. F. also acknowledges the hospitality of the Institute for Theoretical Physics at the University of California, Santa Barbara (NSF grant PHY 99-07949) where this work has evolved. We thank M. Pérault for providing us with his LVG code. The Caltech Submillimeter Observatory is funded by NSF grant AST 99-80846.

\section{REFERENCES}

Bertoldi, F., \& McKee, C. F. 1992, ApJ, 395, 140

Bonazzola, S., Falgarone, E., Heyvaerts, J., Pérault, M., \& Puget, J.-L. 1987, A\&A, 172, 293

Bonazzola, S., Pérault, M., Puget, J.-L., Heyvaerts, J., Falgarone, E., \& Panis, J.-F. 1992, J. Fluid Mech., 245, 1

Boulares, A., \& Cox, D. P. 1990, ApJ, 365, 544

Cadot, O., Douady, S., \& Couder, Y. 1995, Phys. Fluids, 7, 630

Dame, T. M., Elmegreen, B. G., Cohen, R. S., \& Thaddeus, P. 1986, ApJ, 305,892

Douady, S., Couder, Y., \& Brachet, M. E. 1991, Phys. Rev. Lett., 67, 983

Falgarone, E., Panis, J.-F., Heithausen, A., Pérault, M., Stutzki, J., Puget, J.-L., \& Bensch, F. 1998, A\&A, 331, 669 (F98)

Falgarone, E., \& Pérault, M. 1987, in Physical Processes in Interstellar Clouds, ed. G. E. Morfill \& M. Scholer (Dordrecht: Reidel), 59

Falgarone, E., \& Phillips, T. G. 1996, ApJ, 472, 191

Falgarone, E., Puget, J.-L., \& Pérault, M. 1992, A\&A, 257, 715

Fiege, J. D., \& Pudritz, R. E. 2000a, MNRAS, 311, 85

2000b, MNRAS, 311, 105

Flower, D. R., \& Pineau des Forêts, G. 1998, MNRAS, 297, 1182

Franqueira, M., Tagger, M., \& Gómez de Castro, A. I. 2000, A\&A, 357, 1143

Gregersen, E. M., \& Evans, N. J., II 2000, ApJ, 538, 260
Hegmann, M., \& Kegel, W. H. 2000, A\&A, 359, 405

Ingalls, J. G., Bania, T. M., Lane, A. P., Rumitz, M., \& Stark, A. A. 2000, ApJ, 535, 211

Joulain, K., Falgarone, E., Pineau des Forêts, G., \& Flower, D. 1998, A\&A, 340, 241

Klessen, R. S., Heitsch, F., \& Mac Low, M.-M. 2000, ApJ, 535, 887

Kulsrud, R., \& Pearce, W. P. 1969, ApJ, 156, 445

Lee, C. W., Myers, P. C., \& Tafalla, M. 1999, ApJ, 526, 788

Lis, D. C., Pety, J., Phillips, T. G., \& Falgarone, E. 1996, ApJ, 463, 623

Mac Low, M.-M. 1999, ApJ, 524, 169

McKee, C. F., Storey, J. W. V., Watson, D. M., \& Green, S. 1982, ApJ, 259, 647

Myers, P. C., \& Lazarian, A. 1998, ApJ, 507, L157

Ostriker, E. C., Gammie, C. F., \& Stone, J. M. 1999, ApJ, 513, 259

Pety, J. 1999, Ph.D. dissertation, Univ. Paris

Porter, D. H., Pouquet, A., \& Woodward, P. R. 1994, Phys. Fluids, 6, 2133

Scoville, N. Z., Yun, M. S., Clemens, D. P., Sanders, D. B., \& Waller, W. H. 1987, ApJS, 63, 821

Solomon, P. M., Rivolo, A. R., Barrett, J., \& Yahil, A. 1987, ApJ, 319, 730

Spitzer, L. 1978, Physical Processes in the Interstellar Matter (New York: Wiley) 
Tafalla, M., Mardones, D., Myers, P. C., Caselli, P., Bachiller, R., \& Benson, P. J. 1998, ApJ, 504, 900

Tagger, M., Falgarone, E., \& Shukurov, A. 1995, A\&A, 299, 940

Ungerechts, H., \& Thaddeus, P. 1987, ApJS, 63, 645
Vázquez-Semadeni, E., Ostriker, E. C., Passot, T., Gammie, C. F., \& Stone, J. M. 2000, in Protostars and Planets IV, ed. V. Mannings, A. P. Boss, \& S. S. Russell (Tucson: Univ. Arizona Press), 3

Ward-Thompson, D., Scott, P. F., Hills, R. E., \& André, P. 1994, MNRAS, 268,276 